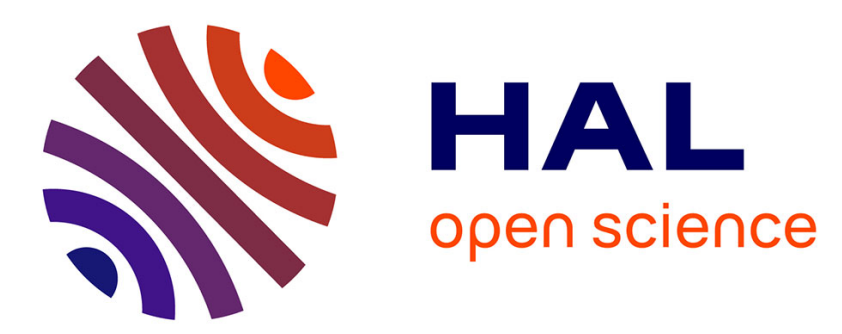

\title{
Les perspectives d'e-Inclusion dans le secteur du handicap mental
}

Véronique Le Chêne, Pascal Plantard

\section{To cite this version:}

Véronique Le Chêne, Pascal Plantard. Les perspectives d'e-Inclusion dans le secteur du handicap mental. Terminal. Technologie de l'information, culture \& société, 2014, Pratiques des outils numériques et relations sociales, 115, pp.11-30. hal-01084277

\section{HAL Id: hal-01084277 \\ https://hal.science/hal-01084277}

Submitted on 16 Jun 2015

HAL is a multi-disciplinary open access archive for the deposit and dissemination of scientific research documents, whether they are published or not. The documents may come from teaching and research institutions in France or abroad, or from public or private research centers.

Copyright

L'archive ouverte pluridisciplinaire HAL, est destinée au dépôt et à la diffusion de documents scientifiques de niveau recherche, publiés ou non, émanant des établissements d'enseignement et de recherche français ou étrangers, des laboratoires publics ou privés. 


\title{
Les perspectives
}

\section{d'e-Inclusion dans le secteur du handicap mental}

\author{
Pascal Plantard, Véronique Le Chêne*
}

\begin{abstract}
Résumé
Les technologies numériques ont envahi notre quotidien et notre vie professionnelle. Leurs usages permettent de nouveaux modes de communication, d'organisation, de production et d'échange. Mais il existe des inégalités numériques qui semblent être le prolongement d'inégalités sociales. Les personnes en situation de handicap mental et/ou psychique, victimes de représentations sociales stigmatisantes, sont confrontées à ces inégalités. Cependant, dans le cadre de leur activité professionnelle, on leur demande d'acquérir des compétences dans les usages de ces technologies, afin de maintenir une compétitivité dans les Établissements et services d'aide par le travail (Esat) qui les emploient. Chargés de mission e-Inclusion au sein des six Esat de l'Adapei 35, nous devions trouver des solutions pour diffuser l'usage des technologies numériques auprès de ces personnes dans le cadre de leurs activités professionnelles. En adaptant les technologies et les méthodes pédagogiques associées lors d'une expérimentation avec des tablettes tactiles auprès de 80 travailleurs, nous avons mis en æuvre une rechercheaction qui avait pour objectif d'étudier les processus d'appropriation personnels, professionnels et institutionnels de ces technologies. Si les participants ont adopté les technologies, ils ont également démontré des capacités et développé de nouvelles compétences.
\end{abstract}

\section{Abstract}

Digital technologies have invaded our lives, professional or not. Using them allow new modes of communication, organization, production and exchange. However digital inequality is a fact and part of it seems to be a natural continuation of social inequality. People with mental and/or psychological disabilities, victims of stigmatizing social representations, are facing

* Courriel : Pascal.Plantard@uhb.fr et veronique.lechene@sfr.fr.

Université Rennes 2 - Place du recteur Henri Le Moal - 35044 Rennes cedex.

Courriel : CREAD et GIS M@rsouin

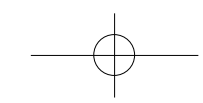


such inequalities. Indeed, in their workplace, they have to acquire skills in the use of these technologies. It's a point of need to maintain competitiveness in Establishments and support services through work (Esat) that employ them. Beeing an e-Inclusion project manager within six Esat of Adapei 35, we had to find solutions to spread the use of digital technologies among these persons in their work environment. By adapting the technologies and teaching methods during a touch pads experiment with 80 workers, we have implemented an action research. The purpose was to study the processes of personal, professional and institutional appropriation of these technologies. Whether participants have adopted the technology, they also demonstrated abilities and developed new skills.

Mots-clés : handicap, technologies numériques, usages, appropriation, eInclusion

Keywords : handicap, digital technologies, uses, appropriation, e-Inclusion

$\mathrm{L}$ es technologies numériques sont aujourd'hui omniprésentes dans notre quotidien et dans notre vie professionnelle. Leurs usages permettent d'autres modes de communication, d'organisation, de production et d'échanges. Toutefois, les études réalisées par le Groupement d'intérêts scientifiques $\mathrm{M} @$ rsouin $^{1}$ nous montrent qu'il existe des inégalités d'accès qui, pour partie, semblent être le prolongement d'inégalités sociales. Les personnes isolées, celles qui ont un faible niveau d'études et un petit revenu, les seniors et les demandeurs d'emplois sont plus nombreux à en être exclus. De plus, au-delà de cette fracture numérique connue et mesurée, "il existe une fracture plus importante qui réside dans la qualité d'utilisation des TICs, dans la capacité à traiter, produire et sélectionner de l'information, dans la capacité d'apprendre en permanence, dans la capacité à jouer un rôle actif dans la société de l'information ${ }^{2}$ ".

Nous ne sommes pas tous égaux devant les technologies et la course au développement des nouvelles fonctionnalités rend l'appropriation des TIC de plus en plus difficile aux personnes déjà exclues du numérique. Sans accompagnement, les effets positifs associés aux usages des TIC ne profitent donc pas à tous.

1 M@rsouin : Môle @rmoricain de Recherche sur la S0ciété de l'information et les Usages d'Internet. Ce groupement d'intérêts scientifiques rassemble des as en sciences humaines et sociales de 11 laboratoires bretons. Ils observent, mesurent et analysent les usages du numérique en Bretagne. M@arsouin. Disponible à partir du : http://www.marsouin.org/ (consulté le 10 février 2014).

2 Charte pour l'inclusion numérique et sociale, (2004), Barcelone. Disponible à partir du : http://charte.velay.greta.fr/ (consulté le 10 février 2014).

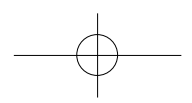


Les personnes en situation de handicap mental et/ou psychique accueillies dans les établissements de l'Adapei ${ }^{3} 35$ sont des «e-exclus ». Lors d'une première immersion réalisée sur une période de 8 mois dans un centre d'hébergement géré par cette association, nous avons observé que les 220 personnes accueillies étaient en situation d'exclusion numérique. Seulement $5 \%$ d'entre elles avaient un ordinateur personnel et $6 \%$ avaient utilisé ponctuellement l'ordinateur lors d'ateliers photos ou vidéo.

Nous avons également observé que certaines personnes, du fait de problèmes d'abstraction, rencontraient des difficultés dans l'usage de la souris et du clavier. Cependant lors d'une évaluation interne réalisée dans cet établissement $34 \%$ des résidents ont exprimé le besoin d'apprendre à utiliser les technologies numériques. Nous avons alors élaboré pour 2011-2012, une première recherche-action qui donnait, à la fois, un accès direct aux TICs et un accompagnement personnalisé aux résidents qui en avaient exprimé le besoin, tout en continuant à étudier leurs usages de ces technologies. Puis, de septembre 2012 à juin 2013, nous avons effectué une recherche-action de plus grande envergure dans les Établissements et services d'aide par le travail (Esat $)^{4}$ de l'Adapei 35 . Au-delà de l'accès direct aux technologies numériques, il s'agissait :

- d'étudier les usages professionnels des ouvriers en situation de handicap mental des Esat,

$\checkmark$ puis d'imaginer les adaptations des dispositifs sociotechniques for-

3. Adapei : Association départementale des amis et parents de personnes en situation de handicap mental de l'Ille-et-Vilaine, créée en 1961 par des parents d'enfants en situation de handicap mental. L'action de cette association de loi 1901 est fondée sur le respect de la dignité des personnes, de leur expression et de leur citoyenneté. Elle recherche la solidarité entre les familles et privilégie l'action col lective. Elle veille à ce que les personnes accueillies soient les auteurs et les acteurs de leur projet. Cela nécessite des rencontres régulières entre les acteurs de la prise en charge de l'usager, les famil les, les représentants légaux et l'usager lui-même afin de définir ses besoins et les moyens à mettre en œuvre pour le suivi des grands axes d'accompagnement : I'habitat, la vie sociale, l'hygiène et la santé, le temps libre, les déplacements, les vacances et la gestion du budget. L'Adapei 35 est membre de la fédération Unapei (Union nationale des associations de parents, de personnes handicapées mentales et de leurs amis) qui fédère 550 associations françaises de représentation et de défense des intérêts des personnes handicapées mentales et de leurs familles. Ces associations régionales, départementales et locales (URAPEI, Adapei, Apei) regroupent 60000 familles, emploient 80000 professionnels et gèrent 3100 établissements qui accompagnent 180000 personnes handicapées.

4. Les Esat sont des établissements médico-sociaux qui proposent aux personnes en situation de handicap mental et/ou psychique une activité à caractère professionnel et un soutien médico-social et éducatif. Pour l' Adapei 35, ils sont répartis sur 6 sites : Saint-Jacques de la Lande, Redon, Dol de Bretagne, Betton, I'Hermitage et Cesson Sévigné. L'association gère aussi des Instituts médico-éducatifs (IME) qui prennent en charge des enfants et des adolescents entre 6 et 20 ans dans l'objectif de faire émerger, de soutenir et de développer des compétences en vue d'une autonomie dans la vie sociale, d'une insertion dans la vie professionnelle et d'une construction de la personnalité. L'association gère également des centres d'habitat qui offrent aux personnes adultes un mode d'habitat adapté à leurs besoins et à leurs souhaits : foyers d'hébergement, résidences pour personnes âgées, hébergement temporaire ainsi que des foyers de vie et des foyers d'accueil médicalisés pour les personnes qui n'ont pas les capacités d'exercer une activité professionnelle. L'ensemble de ces établissements permettent d'accueillir et d'ac compagner les personnes en situation de handicap mental ou maladie psychique tout au long de leur vie. 
més par les instruments numériques et les méthodes d'accompagnement pédagogiques associées,

- et enfin, d'expérimenter ces adaptations dans l'objectif de proposer une méthodologie d'introduction de ces technologies auprès des professionnels et des salariés des Esat.

L'objectif de cette nouvelle recherche-action est bien l'e-Inclusion des salariés en situation de handicap mental. Elle dépasse les questions d'usages personnels des technologies numériques pour s'intéresser aux usages favorisant l'insertion professionnelle.

Dans un premier temps, nous présenterons le contexte de notre recherche-action. Ensuite, nous nous arrêterons sur la recherche elle-même et les méthodes utilisées : tout d'abord nos méthodes d'accompagnement puis nos méthodes de recueil de données dans une approche anthropologique. Enfin, nous exposerons nos résultats et conclusions à savoir les premiers effets de la mise en œuvre d'un processus d'appropriation des technologies numériques par les travailleurs en situation de handicap mental et/ou psychique.

\section{Contexte}

Les Esat de l'Adapei 35 accueillent aujourd'hui 780 travailleurs qui sont majoritairement $(70 \%)$ en situation de handicap mental. Les origines du handicap mental sont diverses et pour $30 \%$ des cas elles restent inconnues. Certaines sont génétiques, d'autres sont accidentelles. Les conséquences du handicap sont différentes selon chaque individu et sont dépendantes du type de déficience, de l'environnement social et familial et des prédispositions de la personne. Certaines auront plus de difficultés à mémoriser les informations, à fixer leur attention, à se repérer dans l'espace et dans le temps, à comprendre les modes d'utilisation des appareils, à maîtriser la lecture ou l'écriture.

La loi du 11 février 2005 pour l'égalité des droits et des chances, la participation et la citoyenneté des personnes handicapées introduit une nouvelle définition qui ne met plus le handicap de la personne en avant mais le fait que c'est la société qui n'est pas adaptée à la personne : "Constitue un handicap, au sens de la présente loi, toute limitation d'activité ou restriction de parti cipation de la vie en société subie dans son environnement par une personne en raison d'une altération substantielle, durable ou définitive d'une ou plusieurs fonctions physiques, sensorielles, mentales, cognitives ou psychiques, d'un polyhandicap ou d'un trouble de santé invalidant ${ }^{5}$ ". Les établissements médico-sociaux que sont les Esat leur offrent donc une compensation du

5 Loi du 11 février 2005, Article 2. Disponible à partir du : www.legifrance.gouv.fr/affichTexte.do?cidTexte $=$ JORFTEXT000000809647\&dateTexte=\&categorieLien=id (consulté le 10 février 2014).

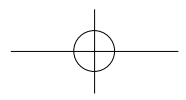


handicap par un accompagnement humain et un environnement de travail adapté à leurs difficultés. Actuellement, le public accueilli dans les établissements de l'Adapei 35 est en pleine évolution avec des nouveaux venus qui souffrent de handicap psychique. Selon l'Unafam 6 , "le handicap psy chique est caractérisé par un déficit relationnel, des difficultés de concentration, une grande variabilité dans la possibilité d'utilisation des capaci tés"7. Les capacités intellectuelles des personnes ne sont pas affectées, mais leur mise en œuvre est complexe. Certaines auront des difficultés à prendre soin d'elles, à établir des relations durables, à se former, à exercer une activité et à organiser une vie sociale et des loisirs. De plus, la prise de médicaments, souvent indispensable, peut affecter leurs capacités intellectuelles de manière irrémédiable.

Entre pairs, dans les établissements spécialisés que sont les Esat, les personnes en situation de handicap mental et/ou psychique sont séparées des non-déficients. Selon Alain Blanc (2006 : 177), cette situation a pour conséquence un isolement des personnes et une méconnaissance du handicap dans notre société. L'éloignement et la méconnaissance de la déficience 8 favorise l'émergence de représentations sociales. Dans une étude sur l'insertion des personnes handicapées mentales dans une communauté sociale, Denise Jodelet (2003) fait ressortir les aspects stigmatisants de ces représentations. Le travailleur en situation de handicap mental est perçu comme instable, irresponsable, dépendant et incapable d'acquérir une qualification.

Ces représentations sont également observables dans les dénominations du handicap. Si les mots « anormal », " incapable », « infirme », « invalide » ne sont plus utilisés dans les discours et textes officiels car entachés de connotations péjoratives, pour Éric Plaisance, " d'autres sonnent plus moderne : handicap et situation de handicap, besoins particuliers ou spéci fiques, trisomie, etc. Mais les mots contribuent toujours à faire des choses et notre vigilance doit s'exercer en permanence " $"{ }^{9}$. Un déficit important de l'estime de soi peut être la conséquence de l'intégration de ces représentations

6. Unafam : Union nationale des familles et amis de malades psychiques créée en 1963, association reconnue d'utilité publique qui accueille, soutien et informe 15000 familles confrontées aux troubles psychiques d'un des leurs. Elle est présente dans 97 départements français grâce à l'action de 2000 bénévoles et d'une vingtaine de salariés répartis entre le siège et les délégations régionales et départementales.

7. Unafam, spécificité du handicap psychique. Disponible sur : www.unafam.org/Specificite-de-I-handicap.html (consulté le 10 février 2014).

8. Le handicap est la limitation dans les possibilités d'interaction d'une personne avec son environnement. II est la conséquence sociale d'une déficience intellectuelle. Selon l'OMS, " la personne ayant une déficience intellectuelle a une capacité plus limitée d'apprentissage et un développement de l'intelligence qui diffère de la moyenne des gens ". Ce n'est pas une maladie, mais un état permanent qui apparaît avant 18 ans. Disponible sur http://informations.handicap.fr/art-handicap-1-3110.php (consulté le 10 février 2014).

9. Plaisance, É. (2009) " Autrement capables. École, emploi, société : pour l'inclusion des personnes handicapées ". Paris : Éditions Autrement, p.13-14. 
stigmatisantes par la personne. Ce déficit touche alors tous les aspects de la vie. C'est ce que nous avons observé lors de notre immersion de dix mois. Les personnes accueillies ont exprimé un manque de confiance en elles quant à leurs capacités à utiliser les technologies numériques : "J'ai la trouille, j'ai peur de pas savoir". "L'ordinateur ce n'est pas pour moi, c'est pour les moniteurs et ceux qui savent lire".

Enfermées dans la déficience et dans des établissements spécialisés, les personnes stigmatisées [Goffman, 1975] entamaient, jusque dans les années 1990, une carrière prédéterminée. Aujourd'hui, leur parcours de vie est moins rectiligne. Les classes pour l'inclusion scolaire (CLIS) en primaire et les unités localisées pour l'inclusion scolaire (ULIS) au collège et au lycée permettent la scolarisation des élèves dans les établissements scolaires ordinaires, même si leur handicap nécessite une organisation pédagogique adaptée. Des détachements « hors les murs $»^{10}$ offrent la possibilité aux travailleurs d'Esat d'exercer une activité professionnelle dans les entreprises du milieu ordinaire tout en bénéficiant d'un accompagnement médico-social. Enfin l'insertion des personnes dans le milieu ordinaire de travail est la mission historique de ces établissements.

Mais l'usage des technologies numériques dans le monde de l'entreprise a transformé les modes de productions et d'organisation. Pour s'insérer les travailleurs d'Esat doivent développer de nouvelles compétences dans les usages de ces instruments. Ces compétences sont également nécessaires pour un maintien dans leur poste en milieu protégé. Les Esat subissent actuellement la disparition de certains marchés de sous-traitance du fait de délocalisations. Ils doivent rechercher de nouveaux contrats. Si de nouvelles potentialités s'offrent à eux avec le développement des productions artisanales, de la filière environnementale (valorisation des déchets et recyclage) et de l'économie numérique, ces activités nécessitent des niveaux de qualification plus élevés et des compétences dans l'usage des technologies numériques. Ces compétences seront un pré-requis sur le marché de l'emploi, même dans le secteur protégé du fait, par exemple, de la numérisation généralisée des machines-outils (exemple : tour numérique) et de la complexification du travail (exemple : gestion de stock informatisée).

De plus, ce sont les clients des Esat qui "fixent la valeur productive du travail et déterminent le marché dans lequel s'inscrira l'activité professionnelle des personnes handicapées mentales"11. Ces donneurs d'ordres ont les

10. Détachement " hors les murs " : la personne en situation de handicap mental en contrat de soutien et d'aide par le travail avec l'Esat peut être mise à disposition d'une entreprise du milieu ordinaire de travail. Dans les Esat de I'ADAPEI 35, ces détachements se font à temps partiels pour que la personne puisse continuer à bénéficier de temps d'accompagnement médico-social et éducatif. Lorsque son détachement se termine, elle revient travailler au sein de l'Esat. Ces contrats passerelles ont pour objectif de favoriser l'intégration des personnes et de faciliter une embauche ultérieure dans le milieu ordinaire. 11. UNAPEI « Travail et handicap » (2011) Livre blanc : UNAPEI, p.20.

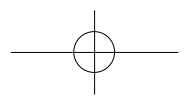


mêmes exigences envers les établissements du secteur protégé qu'envers les entreprises du milieu ordinaire en termes de qualité, de délais et de coûts. Les Esat doivent donc avoir des usagers productifs. Ce qui est paradoxal quand on sait que ces établissements accueillent des personnes avec des déficiences. Ces contraintes vont donc inciter les établissements à conserver les travailleurs les plus productifs pour maintenir leur productivité. Cela va à l'encontre de la mission historique d'accompagnement vers une insertion en milieu ordinaire de travail pour les personnes les plus autonomes et productives. Le risque est de voir apparaître une diminution du soutien médicosocial et éducatif au profit de la production, une sélection des personnes en fonction de leur rentabilité et une pression croissante sur les travailleurs.

Ce contexte fait émerger un premier questionnement : dans un objectif de professionnalisation, les travailleurs d'Esat doivent acquérir des compétences dans les usages des technologies numériques, mais comment développer ces nouvelles aptitudes auprès de ces personnes déficientes intellectuelles, parfois encore stigmatisées et en manque de confiance en elles ?

\section{La recherche EIHM}

Le projet EIHM ${ }^{12}$ a pour objectif de donner un accès direct aux technologies numériques pour les travailleurs en situation de handicap mental et/ou psychique des Esat et d'étudier leurs processus d'appropriation personnels, professionnels et institutionnels.

Ce projet fait suite à une première recherche-action réalisée en 20112012 avec ce même public dans un foyer d'hébergement. Elle avait pour objectif de donner accès à ces technologies aux résidents de cet établissement et de trouver des méthodes pour qu'ils se les approprient dans un objectif d'insertion sociale. En fonction des difficultés des personnes, il avait été nécessaire d'adapter les méthodes pédagogiques ainsi que les technologies. L'écran tactile avait été le support de cette première recherche-action. S'il a permis l'accès à l'ordinateur, son utilisation a également révélé des capacités de concentration, d'adaptation, d'apprentissage et de compréhension des résidents. Mais pour certaines personnes, l'utilisation de cet environnement était difficile du fait de sa position verticale (pas de point d'appui).

Le projet de recherche-action EIHM, lui, est fondé sur l'utilisation de tablettes tactiles mobiles que l'on peut poser sur un plan horizontal. Ces tablettes ont été mises à disposition par le Centre de recherche sur l'éducation, les apprentissages et la didactique (CREAD) de l'Université Rennes 2. Au-delà des pratiques personnelles, l'objectif de ce projet est aussi d'élargir les questions de recherche à l'insertion professionnelle.

À la suite des travaux M@rsouin sur les "exclus" du numérique, nous postulons l'importance du changement des représentations quant à l'utilisation

12. EIHM : E-inclusion dans le secteur du handicap mental.

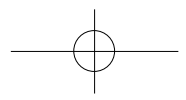


des technologiques numériques par les travailleurs en situation de handicap mental : celles des travailleurs, des moniteurs d'ateliers et des proches des personnes. Pour Anne-Marie Hugon et Claude Seibel, les recherches-actions sont des "recherches dans lesquelles il y a une action délibérée de transfor mation de la réalité [...] et produire des connaissances concernant ces trans formations $\gg^{13}$. Cette nouvelle recherche-action aura donc pour objectif une transformation des représentations sociales concernant les relations entre le handicap mental et les usages du numérique ainsi qu'un recueil de données qualitatives sur les effets de ces transformations pour les travailleurs d'Esat.

Nos questions de recherche sont les suivantes :

- L'usage des tablettes tactiles par les travailleurs en situation de handicap mental et/ou psychique permet-il le « dévoilement $»^{14}$ de leurs capacités affectives, cognitives et relationnelles ?

- En portant à la (re)connaissance des encadrants ces capacités « dévoilées », seront-ils incités à dépasser les représentations qu'ils ont, à la fois sur les personnes et sur le numérique (renforcement) ? À partir de ces transformations de représentations, les encadrants mettront-ils en place des pratiques numériques nouvelles dans les ateliers ?

- La valorisation de ces capacités par l'environnement social, en particulier les encadrants, interviendra-t-elle sur l'estime de soi des personnes, sur leur confiance en elles et favorisera-t-elle de nouvelles sociabilités (lien)?

\section{Méthodologies}

La recherche-action a été coconstruite entre trois principaux acteurs : les cadres de l'Adapei 35 et des Esat, un chercheur spécialiste de l'eInclusion et une jeune chercheuse déjà en immersion dans un foyer d'hébergement de l'Adapei 35 depuis 2011.

Dans un premier temps, il a été nécessaire d'initier la phase d'apprivoisement mutuel [Plantard, Trainoir, 2012] dans ce nouveau terrain des Esat. Pour l'étayer, nous avons réalisé un état des lieux des pratiques numériques des travailleurs d'Esat. De nombreuses personnes ne sachant ni lire, ni écrire, il n'a pas été possible (ni souhaitable) de réaliser un questionnaire. Il était nécessaire de mettre les personnes en confiance pour l'émergence de la parole. Nous avons fait le choix d'aller à leur rencontre directement sur leur poste de travail. Nous avons recensé/observé les pratiques numériques dans les ateliers de travail auprès de 600 travailleurs des Esat de l'Adapei 35. Ce recensement a été effectué en observant les pratiques effectives, puis en les

\footnotetext{
13. Hugon, M-A., Seibel, C. (1988), "Recherches impliquées, recherches action le cas de l'éducation ", De Boeck université : Éditions universitaires, p. 13.

14. En référence aux trois " pouvoirs " des usages : dévoilement, renforcement et lien (Plantard, 2011).

18

] terminal $n^{\circ} 115[$
}

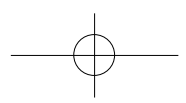


codant de manière à produire des indicateurs statistiques. Nous avons aussi recueilli, par entretiens non directifs, les attentes et les demandes concernant le numérique. Cela s'est déroulé sous la forme d'une immersion quotidienne dans les Esat sur une période longue (cinq mois). La méthodologie employée est celle de l'observation participante [Lapassade, 1991]. La chercheuse en immersion observait les pratiques numériques dans le quotidien des travailleurs des Esat.

Dans un deuxième temps, sur les cinq mois suivants, la recherche s'est développée dans une action spécifique. En position de « médiation numérique » [Plantard, 2013], c'est-à-dire d'accompagnement individualisé ou en petit groupe de pratiques numériques, nous avons réalisé des ateliers « d'apprivoisement $»$ de la tablette tactile auprès de 80 travailleurs. Nous avons choisi des personnes qui n'ont pas souvent été mises en avant, de part leur activité professionnelle ou extraprofessionnelle, des personnes en déficit d'estime de soi ainsi que d'autres éloignées des pratiques numériques.

Nous avons également choisi des personnes insérées dans la démarche de reconnaissance des acquis de l'expérience professionnelle dans le cadre du dispositif « Différent et compétent ${ }^{15}$. Ces personnes doivent réaliser un dossier montrant leurs compétences dans l'exercice d'une activité professionnelle. La validation de ce dossier par des professionnels du monde de l'entreprise apporte une reconnaissance spécifique aux travailleurs d'Esat : la reconnaissance des acquis de l'expérience (RAE).

Pour la réalisation de ce dossier, certains utilisent l'ordinateur, d'autres rencontrant des difficultés dans l'usage de ces instruments le font de manière manuscrite. L'acquisition de compétences dans les usages des technologies numériques peut être vue comme un élément facilitateur à la réalisation de ces dossiers. Lors de cette expérimentation avec les tablettes tactiles, nous avons appris à connaître les personnes, puis à les reconnaître au travers de leurs capacités.

Enfin nous sommes entrés dans un processus de reconnaissance mutuelle : «Reconnaître l'autre comme un vis-à-vis de même tissu que soi et donc aussi se reconnaître en l'autre » ${ }^{16}$. C'est, selon Henri-Jacques Stiker, ce cycle de la reconnaissance qui aboutit à la singularité. La personne ne fait plus partie de la catégorie socialement construite des handicapés, mais elle est une personne unique et spécifique avec des besoins, des capacités et des difficultés. Cette reconnaissance a pour objectif un renforcement de l'estime de soi. Pour créer une relation de confiance vis-à-vis de la technologie, nous avons choisi une approche ludique afin de dédramatiser les difficultés et les

15. Différent et compétent. Disponible sur www.tugdual-ruellan-communication.eu/public/DIFFE RENT_ET_COMPETENT/D_C_RESEAU/D_C.LETTRE/2013.11.01.DetC-LETTRE_n-3.pdf (consulté le 10 février 2014).

16. Stiker, H-J. (2011) " II suffit d'une petite différence " in Piot, M. Handicap, estime de soi, regard des autres. Paris : l'Harmattan, p. 144

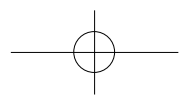


appréhensions. Nous avons été attentifs à les préserver des situations d'échec. Nous avons également choisi des méthodes pédagogiques actives afin que les personnes deviennent actrices de leurs apprentissages et qu'elles découvrent la tablette tactile par expérimentation et tâtonnement en fonction de leurs centres d'intérêts et de leurs besoins.

On retrouve dans cette pédagogie non directive certains des invariants pédagogiques énoncés par Célestin Freinet [1969]. S'ils concernent l'enfant, nous pensons qu'ils sont transposables aux personnes accompagnées lors de cette recherche-action. L'accompagnement non directif renvoie à l'invariant 4 : "Nul - l'enfant pas plus que l'adulte - n'aime être commandé d'autorité ". Le centrage sur les besoins de la personne répond à l'invariant 7 : "Chacun aime choisir son travail, même si ce choix n'est pas avantageux ». L'approche ludique et la pédagogie de la réussite concordent avec l'invariant 10bis : " Tout individu veut réussir. L'échec est inhibiteur, destructeur de l'allant et de l'enthousiasme ». L'approche expérimentale correspond aux invariants 11 à 13 : "La voie normale de l'acquisition n'est nullement l'observation, l'explication et la démonstration processus essen tiel à l'école, mais le tâtonnement expérimental, démarche naturelle et uni verselle ».

Nous avons également mis en œuvre des méthodes d'empowerment ${ }^{17}$ en vue d'une autonomie dans les usages. Pour cela, nous avons accompagné les personnes dans la prise d'initiatives et l'expression de soi.

Dans un troisième temps, nous avons réalisé des tests de psycho-ergonomie cognitive au sein du Laboratoire Loustic avec sept travailleurs d'Esat. Nous avons choisi des personnes qui exprimaient régulièrement leurs impressions, leurs ressentis : que ce soit une expression verbale ou non verbale, que les personnes aient des difficultés d'élocution ou pas. L'objectif de cette expérimentation complémentaire en laboratoire était d'observer encore plus finement les aspects émotionnels dans l'appropriation des technologies numériques, le renforcement de l'estime de soi au travers de l'usage de la tablette tactile, ainsi que les transformations des modes d'apprentissage. Ces tests ont fait l'objet de captations vidéo qui ont été ensuite analysées.

Parallèlement, nous avons réalisé une collecte de données qualitatives et contextuelles. L'objectif était de connaître l'environnement des personnes et de recueillir, dans la mesure du possible, des bribes de leurs histoires de vie. Soixante personnes se sont exprimé lors des ateliers avec les tablettes tactiles. L'émergence de la parole a été grandement facilitée par la pro x i $\mathrm{m}$ i t é avec les pairs et le cadre familier, ce qui n'a pas été le cas lors d'entretiens exploratoires formalisés avec deux travailleurs d'Esat. La situation d'entretien

17. Cette notion n'a pas vraiment de traduction en français. Serge Proulx parle de " puissance d'agir " (Proulx, 2012). Pascal Plantard parle de processus " d'appropriation-autonomisation-capacitation " (Plantard, 2011).

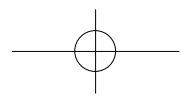


individuel semi-directif est trop proche des situations d'entretiens professionnels avec les psychologues ou les travailleurs sociaux des Esat, ce qui ne facilite pas la libération de la parole. Pour les personnes en situation de handicap, cette méthodologie plus classique a été abandonnée au profit d'une collecte longitudinale en situation d'observation participante lors de l'expérimentation avec les tablettes tactiles. Les entretiens semi-directifs ont été utilisés avec les encadrants des Esat, particulièrement les moniteurs d'ateliers.

\section{Résultats}

Nous avons constaté que les travailleurs des Esat de l'Adapei 35 utilisent peu les technologies numériques dans le cadre des ateliers de travail : $10 \%$ des 600 travailleurs enquêtés les utilisent pour du traitement de texte, des saisies de données dans un tableur ou des scans de codes-barres. Parallèlement à ce résultat, 46,6\% de l'ensemble des personnes rencontrées ont des usages personnels de l'ordinateur et d'Internet (contre $79 \%$ des foyers bretons en 2012) (18 $^{18}$ jouer pour $81 \%$ d'entre elles, effectuer des recherches sur Internet pour $54 \%$, écouter de la musique pour $19 \%$, maintenir les acquis scolaires pour $18 \%$, utiliser une messagerie pour $18 \%$, gérer des photos pour $14 \%$ et utiliser les réseaux sociaux pour $9 \%$.

Nous avons également constaté une forte motivation : $44 \%$ des personnes rencontrées souhaitent apprendre à utiliser l'ordinateur et $25 \%$ souhaitent approfondir leurs connaissances. Il est également important de noter que 9,6\% des personnes sont intéressées par l'idée d'une reconnaissance « académique » de leurs compétences numériques. Les attentes des travailleurs d'Esat concernent principalement le maintien des acquis scolaires, les usages d'Internet et la gestion de la messagerie électronique. Enfin, $22 \%$ ne souhaitent pas utiliser l'ordinateur.

Du côté des moniteurs d'ateliers ${ }^{19}$, les attentes concernent la mise à disposition d'un ordinateur avec une connexion à Internet pour chaque atelier afin de favoriser l'ouverture sur l'extérieur des travailleurs. Ils aimeraient que les personnes développent des aptitudes à utiliser les logiciels de traitement de texte et de tableur pour réaliser des fiches clients, des suivis de production ou de la gestion de stocks. Pour eux, l'objectif prioritaire reste la professionnalisation. Les attentes de l'institution sont de répondre à un besoin croissant de développement des compétences des travailleurs avec quatre

18. OPSIS : Laboratoire statistique du groupement $M @$ rsouin. Usages du numérique en Bretagne, Repères 2012. Disponible à partir du : www.marsouin.org/spip.php?article489 (consulté le 10 février 2014).

19. Le moniteur d'atelier est un travailleur social qui encadre généralement une douzaine de personnes dans un atelier de production ou de prestation de service qu'il gère et organise. Il a également pour mission d'accompagner les personnes en fonction de leurs difficultés. II doit adapter les postes de travail, déceler les potentialités, favoriser l'évolution professionnelle et le bien-être des personnes, les guider vers une acquisition de nouveaux savoirs et les accompagner vers une autonomie dans le travail.

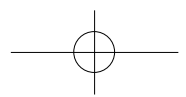


objectifs : le maintien dans le poste, la productivité, l'adaptation aux nouvelles conditions de travail et l'insertion en milieu ordinaire.

Ce dernier objectif concerne les personnes qui le peuvent et qui le souhaitent tout en sachant que ces insertions sont très marginales. Le taux de sortie des travailleurs vers le milieu ordinaire est de 0,25\% dans les Esat de l'Adapei 35. On retrouve dans la notion d'insertion en milieu " ordinaire », l'idée que la personne doit s'adapter au milieu du travail. "L'insertion est essentiellement pensée comme un dispositif s'adressant à des personnes à normaliser en vue d'une adaptation à la vie professionnelle et sociale $»^{20}$. C'est une contradiction par rapport à la loi de 2005 qui met en avant les nécessaires évolutions et adaptations de la société à la personne handicapée qui renvoie à l'inclusion sociale.

Nous définissons l'inclusion sociale comme l'ensemble des interactions endogènes (inclusives) que les personnes entretiennent avec les systèmes sociaux (Luhmann, 2010). C'est le contraire de l'exclusion sociale qui concerne les interactions exogènes. Le schéma suivant (Fig. 1) illustre bien les différences entre les processus d'exclusion, de ségrégation, d'intégration et d'inclusion :

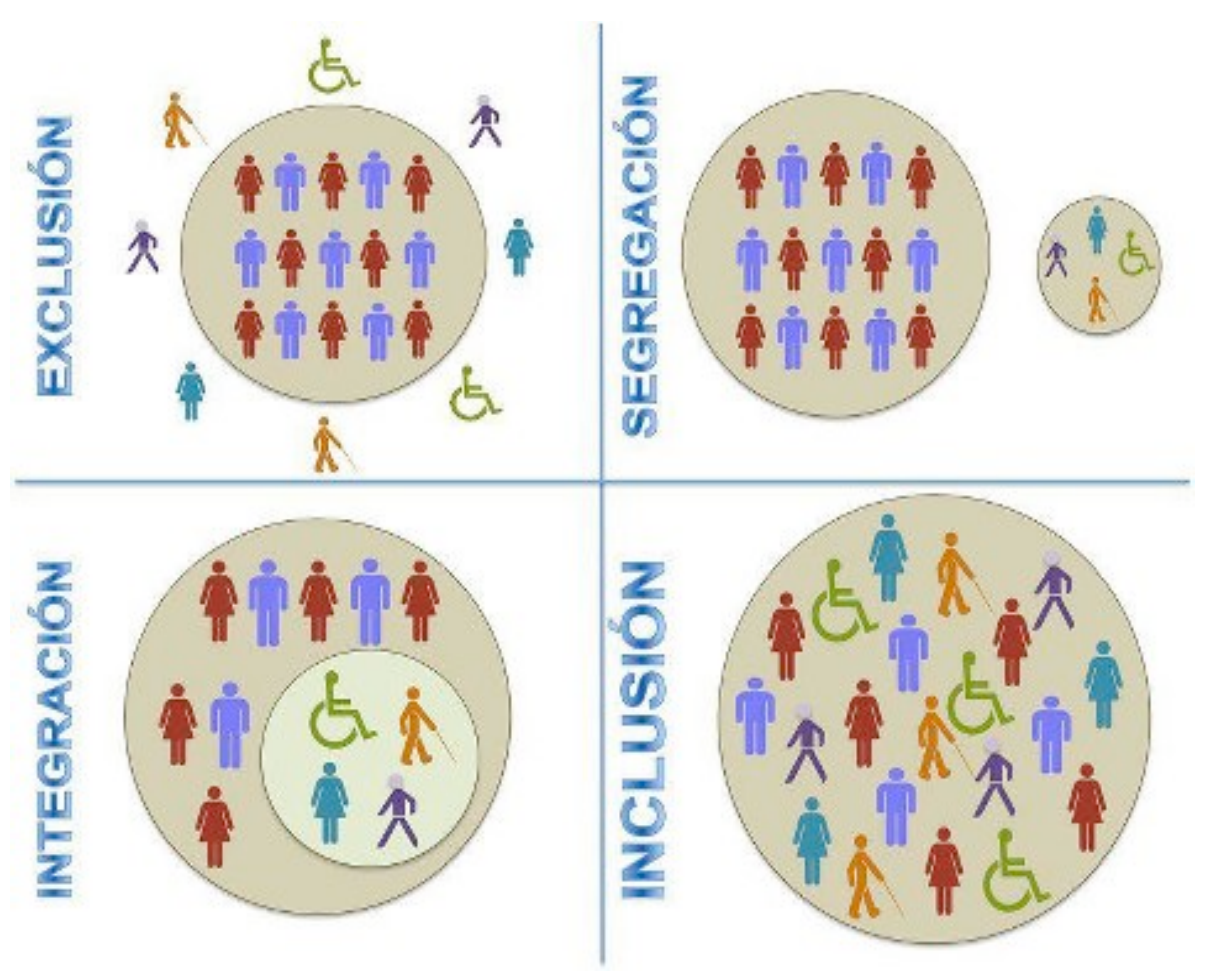

Figure 1 : Source : Mariano Santiago, 2012, Université de Buenos Aires.

20. Loriol, M, (1999). Qu'est-ce que l'insertion sociale ?, Paris : L'Harmattan, p.11.

22

] terminal $\mathrm{n}^{\circ} 115$ [

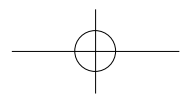


Dans le même sens, l'e-Inclusion peut être définie comme l'ensemble des interactions endogènes (inclusives) que les usagers entretiennent avec les systèmes sociotechniques numériques. Au niveau scientifique, cela conditionne une démarche de recherche dialectique autour de l'identification des nouvelles exclusions provoquées par les technologies numériques, en articulation avec des travaux sur la modélisation des nouvelles médiations numériques qui luttent contre ces exclusions. L'e-Inclusion concerne donc toutes les actions, de la pratique individuelle à la politique publique, qui luttent contre la fracture numérique, même si cette notion est beaucoup plus politique que scientifique.

À l'inverse, la conceptualisation de l'e-Inclusion peut permettre de sortir de l'injonction idéologique du " tout numérique ", ou d'en limiter les effets, dans la perspective de l'interactionnisme symbolique de l'école de Chicago (Blumer, Becker, Goffman...). D'abord l'e-Inclusion s'arrime à l'inclusion sociale du moment, ce qui lui fixe un contexte socio-historique limitant les effets de généralisation idéologique. Ensuite, le sens inclusif que donnent les usagers à un dispositif socio-technique dépend de la norme d'usage en cours. L'usage est défini comme un ensemble de pratiques socialisées, c'est donc à partir des interactions avec les autres usagers que les personnes en situation de handicap vont se représenter, puis s'approprier tel ou tel instrument technologique.

Sur les 80 personnes qui ont participé aux ateliers d'appropriation des tablettes tactiles sur une durée de cinq mois (chaque personne a participé à six ateliers d'une heure trente), 38 n'avaient aucune pratique numérique. Cependant, 53 personnes ont utilisé la tablette sans difficulté, 18 personnes ont eu besoin d'apprendre à utiliser la fonction tactile et seulement 9 personnes ont rencontré des difficultés. Elles ont présenté des capacités de concentration $^{21}$ (69 personnes), des capacités de mémorisation ${ }^{22}$ (67), et des capacités de compréhension ${ }^{23}(60)$.

Aujourd'hui, 16 personnes sont autonomes dans l'utilisation de cette technologie. Sur les trois capacités « concentration / mémorisation / compréhension » avec une moyenne de 65/80, on constate un véritable dévoilement des capacités cognitives. Au travers de l'approche ludique, les personnes ont réussi à mettre en place un raisonnement logique. Elles ont compris les consignes et mis en œuvre une méthode singulière pour répondre à cette consigne et atteindre, par exemple, les objectifs des jeux.

Les ateliers ont aussi permis la mise en place d'une relation de confiance entre les personnes et la chercheuse qui a réalisé l'expérimenta-

21. Rester concentré sur toute la durée des ateliers d'expérimentation, soit une heure trente.

22. Mémoriser les procédures pour allumer la tablette tactile, ouvrir une application, progresser dans les niveaux des jeux et intégrer des photos dans une application de mise en page.

23. Comprendre les consignes d'utilisation de la tablette tactile.

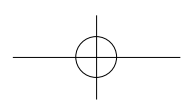


tion : " On est bien avec vous. Moi je suis heureux. J'apprends des choses et on discute un petit peu. C'est bien ", mais également face à l'environnement sociotechnique : "Ça m'a ouvert l'esprit à quelque chose que je ne connaissais pas avant. Au début, on ne sait pas trop comment manipuler, mais c'était pas trop dur pour moi "».

Le tâtonnement expérimental a dédramatisé les appréhensions et renforcé l'estime de soi des personnes. En découvrant le fonctionnement de la tablette par essais-erreurs, les personnes ont pris conscience de leur capacité à réussir par elles-mêmes. "En présence de ces éléments matériels des cul tures numériques, chacun renforce ses comportements de confiance ou de défiance. Pour les accidentés du narcissisme, les personnes en mésestime d'elles-mêmes, la confiance envers les TIC amorce un mouvement de recom position identitaire ${ }^{24}$. C'est le pouvoir de renforcement des usages des technologies numériques. Nous sommes passés d'un discours exprimant des craintes : "J'ai la trouille, j'ai peur de pas savoir » à l'expression d'une grande satisfaction : "Yes, j’ai réussi ! J’ai réussi ! Punaise ! ", " Super, c'est cool, je suis heureux ! ». Les émotions sont primordiales dans l'acceptation des technologies. Elles traduisent le changement de représentation de soi et elles favorisent l'expression.

En confiance avec les chercheurs, les personnes se sont exprimé lors des ateliers et ont dévoilé des histoires de vie. "Dans le contexte de l'insertion, ces pratiques technologiques remplies d'affects ouvrent un dialogue, pas tou jours verbal, où la personne en souffrance en vient à se dévoiler. Le pouvoir de dévoilement ne fonctionne pas isolé, il lui faut une oreille, un accompa gnateur $»^{25}$.

Pour que les encadrants prennent conscience des compétences numériques des participants à l'expérimentation, ces derniers ont présenté à leurs collègues et à leurs moniteurs leurs aptitudes à utiliser les tablettes tactiles. La reconnaissance par les pairs a renforcé l'estime de soi des personnes et a modifié leur rapport au savoir. Ils se sont mis dans la position de celui qui transmet le savoir en présentant leurs aptitudes et en expliquant la manière d'utiliser la technologie. Selon Alain Blanc, "le groupe des pairs élabore une culture collective basée sur une communauté d'expérience dont la défi cience est le socle. Les pairs se parlent, comparent leurs activités et parfois entrent en compétition les uns vis-à-vis des autres [...] La pair-émulation constitue une dynamique socialisatrice $»^{26}$.

Ces échanges ont dévoilé et renforcé leurs capacités relationnelles, même pour les personnes rencontrant des difficultés d'élocution qui ont souhaité s'exprimer. Le désir de reconnaissance par les pairs a renforcé le pouvoir de lien entre les personnes et la présentation des aptitudes à s'approprier

24. Plantard, P. (2011) Pour en finir avec la fracture numérique. Limoges : Fyp, p. 24. 25. Plantard, P. (2001) Op cité, p. 26.

26. Blanc, A. (2006) Le handicap ou le désordre des apparences. Paris : Armand Colin, p. 221.

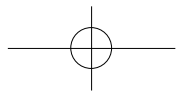


cette nouvelle technologie a attesté de l'appartenance à un autre groupe que celui des " handicapés », celui des usagers du numérique. En effet, " notre profond désir identitaire d'être reconnu par un groupe et de partager avec les autres trouve dans les usages des TIC de nouveaux territoires où tenter l'affranchissement, le changement de groupe et le retour à la norme, même pour les plus marginaux $»^{27}$.

Cette présentation a également changé les représentations des moniteurs d'ateliers. Certains d'entre eux pensaient que les technologiques numériques ne seraient pas accessibles aux personnes qu'ils accompagnent, du fait de l'illettrisme et des déficiences. Ils ont été surpris de ce que les usagers ont réalisé avec les tablettes : en termes de production de documents et d'expression de soi, mais également en termes de concentration, de mémorisation, de repérage spatio-temporel, de capacité à faire un choix et d'initiative. Ils ont pris conscience que l'usage de ces technologies était très valorisante pour les personnes : "Pour une fois, ils ne sont pas à la ramasse! ». Pour favoriser une transformation plus large des représentations autour du handicap mental et du numérique, des articles ont été rédigés dans le magazine national de 1'UNAPEI ${ }^{28}$, et dans les deux principales revues spécialisées du travail social : $\mathrm{TSA}^{29}$ et $\mathrm{ASH}^{30}$.

La valorisation des capacités à s'approprier les technologies numériques va être renforcée par la mise en place d'un partenariat entre l'Adapei 35 et la région Bretagne. L'objectif sera de valider les compétences aux usages de base de ces technologies et d'Internet au travers du Visa Internet Bretagne (certification de compétences numériques de Bretagne). Elle viendra s'ajouter à une reconnaissance personnelle, à un renforcement de l'estime de soi ainsi qu'à une reconnaissance sociale et professionnelle attestée par le changement de regard des pairs et des encadrants.

\section{Conclusion}

Les résultats de cette recherche montrent que la majorité des participants à l'expérimentation ont adopté et accepté les tablettes tactiles car ils en ont perçu l'utilité, les avantages qu'ils pouvaient en retirer et une facilité d'usage. En ayant accès à cette technologie, en apprenant à la maîtriser et en produisant des documents, les personnes sont entrées dans un processus d'appropriation de l'instrument. Mais celui-ci ne sera effectif que lorsque ces pratiques seront intégrées dans leur vie quotidienne et professionnelle.

27. Plantard, P. (2001) Op cité, p. 28.

28. Salignat, M. (2013) « Comment les personnes déficientes intellectuelles se sont-elles emparées du Net? " Le journal de I'UNAPEI. Vivre ensemble, $\mathrm{n}^{\circ} 113, \mathrm{p} .6-11$.

29. Vion, A. (2012) Les travailleurs sociaux tâtonnent sur le Web, TSA, ${ }^{\circ} 34$, p. 15-19.

30. Sédrati-Dinet, C. L'accès au numérique, un défi pour les personnes handicapées, ASH. n 2819-2820, p. 36-39.

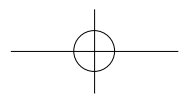


Ces résultats vont influencer directement les conditions de vie et de travail des salariés des Esat de l'Adapei 35 par une transformation de leurs environnements sociotechniques portée par une évolution des représentations que les autres et eux-mêmes ont de leurs capacités numériques, donc cognitives et sociales.

Nous émettons l'hypothèse que le processus d'appropriation du numérique par les travailleurs d'ESAT entraînera une transformation de l'organisation de ces établissements. En effet, si la reproduction des procédures et les méthodes répétitives sont actuellement courantes dans les pratiques professionnelles, nous avons démontré que les personnes en situation de handicap mental et/ou psychique sont parfaitement capables de s'approprier des méthodes actives d'apprentissage (libre choix, pédagogie de la réussite, tâtonnement expérimental, apprentissage entre pairs...) qui les placent dans un processus d'empowerment et favorise l'acquisition d'une part d'autonomie. La prise en compte des souhaits et des demandes des personnes place également les établissements dans un dispositif d'innovation sociale et ascendante [Plantard, 2013]. Nous émettons également l'hypothèse que ce processus d'appropriation transforme les interactions entre les différents acteurs des établissements. Il induit un changement dans les rapports de pouvoir. Les moniteurs d'atelier ne sont plus dans une position unique de transmission d'un savoir descendant. Certains d'entre eux doivent être formés aux usages des technologies numériques. Cette transformation de l'organisation de l'institution est un des facteurs innovants de cette recherche-action. Et si les méthodes pédagogiques utilisées ne sont pas nouvelles, leur mise en œuvre dans les Esat est également innovante.

Au regard des aptitudes et des capacités dévoilées par les usagers lors de l'expérimentation avec les tablettes tactiles, les directeurs d'Esat de l'Adapei 35 souhaitent mettre en place des actions à court terme.

Dans un premier temps, les établissements vont acquérir plusieurs dizaines de tablettes tactiles et les utiliser pour la réalisation des dossiers de reconnaissance des acquis mis en place au travers du dispositif « Différent et compétent ». Une comparaison a été réalisée lors de l'expérimentation de ce dispositif avec une monitrice de soutien : les personnes ont plus de facilité à réaliser ces dossiers avec les tablettes tactiles qu'avec les ordinateurs de bureau.

Dans un deuxième temps, il est prévu de former tous les travailleurs d'Esat, dans le cadre du soutien éducatif, afin de généraliser l'usage de l'internet mobile. Enfin, dans un troisième temps, il est prévu de rechercher des applications spécifiques pour le travail en atelier à destination des travailleurs. Lorsque ces étapes seront effectives au sein des établissements, nous pourrons véritablement identifier une démarche d'appropriation des instruments technologiques par les usagers que Serge Proulx définit comme « le processus d'intériorisation progressive de compétences techniques et cogni-

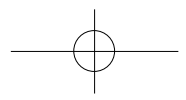


tives à l'œuvre chez les individus et les groupes qui manient quotidiennement ces technologies $»^{31}$.

L'Adapei 35 souhaite élargir le projet e-Inclusion à l'ensemble des établissements du département et développer l'accessibilité aux technologies numériques au sens large : généralisation de la formation des travailleurs d'Esat ainsi que la formation des professionnels (moniteurs d'atelier, éducateurs...) aux usages de ces technologies et aux méthodes pédagogiques actives. Un groupe de travail départemental, en partenariat avec l'université Rennes 2 (CREAD-M@rsouin), élabore actuellement un plan d'action pour la période 2014/2016.

Le premier objectif est de mettre en place une action transversale sur l'ensemble des secteurs de l'association : enfance, travail et hébergement. Un plan de financement pluriannuel est aussi en cours de constitution avec des partenaires inhabituel pour l'Adapei, liés au financement de la recherche ou au numérique. Cette mission servira de support à un nouveau travail de recherche dans le cadre d'une thèse de doctorat sur les effets de ces transformations sociotechniques sur les conditions de vie, de travail et d'insertion des personnes en situation de handicap mental et/ou psychique et sur les institutions qui les accompagnent.

31. Proulx S. (2005) " Penser les usages des TIC aujourd'hui : enjeux, modèles, tendances " in Vieira L., Pinède, N. Enjeux et usages des TIC. 


\section{RÉFÉRENCES}

BLANC, A. (2006) Le handicap ou le désordre des apparences. Paris : Armand Colin

CHAMBAT, P. (1994) Usages des technologies de l'information et de la communication : évolution des problématiques. Disponible à partir du : http://revues.mshparisnord.org/lodel/disparues/docannexe/file/451/vol6_n3_article3.pdf

FREINET, C. (1994) Euvres pédagogiques. Tome 2. Édition établie par Madeleine Freinet. Paris : Seuil

GOFFMAN, E. (1975) Stigmates : les usages sociaux des handicaps, Paris : Éditions de Minuit.

HUGON, M-A., SEIBEL, C. (1988) Recherches impliquées, recherches action le cas de l'éducation, De Boeck université : Éditions universitaires

JODELET, D. (2003) « Représentations sociales de la maladie mentale et insertion des malades mentaux ", in Jean-Claude Abric, Exclusion sociale, insertion et prévention. ERES " Hors collection ", p. 97-113

LORIOL, M. (1999) Qu'est-ce que l'insertion sociale ?, Paris : L'Harmattan

PLAISANCE, É. (2009) Autrement capables. École, emploi, société : pour l'inclusion des personnes handicapées. Paris : Éditions Autrement

PLANTARD, P. (2013) Éducation à la technologie et au numérique. La gaité lyrique. Disponible à partir du : http://www.gouvernement.fr/gouvernement/rencontre-education-a-la-technologie-et-au-numerique-pascal-plantard-1

PLANTARD P. (2013) “ Usages et médiation numérique " In Recherches sur la société du numérique et ses usages - M@rsouin, N³, Paris : L'Harmattan

PLANTARD, P. ET TRAINOIR, M. (2012) “ Contribution à l'anthropologie des usages du numérique " in Recherches sur la société du numérique et ses usages - M@rsouin, $\mathrm{N}^{\circ} 2$, Paris : I'Harmattan. pp. 175-195

PLANTARD, P. (2011) Pour en finir avec la fracture numérique. Limoges : Fyp

PROULX, S. (2012) La puissance d'agir des citoyens dans un monde fortement connecté. Conférence d'ouverture, Colloque d'Agadir, 4-6 avril 2012

PROULX S., JAURÉGUIBERRY, F. (2011) Usages et enjeux des technologies de communication, ERES Poche - Société

PROULX, S. (2005) "Penser les usages des TIC aujourd'hui : enjeux, modèles, tendances " in Vieira, L., Pinède, N. Enjeux et usages des TIC, Bordeaux : Presses universitaires de Bordeaux

STIKER, H-J. (2011) " II suffit d'une petite différence " in Piot, M. Handicap, estime de soi, regard des autres. Paris : I'Harmattan

Travail et handicap (2011) Livre blanc : UNAPEI

\section{Sites Internet :}

Adapei 35. Disponible à partir du : www.adapei35.com.

Charte pour l'inclusion numérique et sociale, (2004), Barcelone. Disponible sur http://charte.velay.greta.fr/ (consulté le 10 février 2014).

Différent et compétent. Disponible sur www.tugdual-ruellan-communication.eu/public/DIFFE RENT_ET_COMPETENT/D_C_RESEAU/D_C.LETTRE/2013.11.01.DetC-LETTRE_n-3.pdf (consulté le 10 février 2014). 
Loi du 11 février 2005, Article 2. Disponible sur www.legifrance.gouv.fr/affichTexte.do?cidTexte=JORFTEXT000000809647\&dateTexte $=\& c$ ateg orieLien=id (consulté le 10 février 2014).

M@arsouin. Disponible sur www.marsouin.org/ (consulté le 10 février 2014).

OPSIS, Repères 2012. Disponible sur www.marsouin.org/spip.php?article489 (consulté le 10 février 2014).

UNAFAM, spécificité du handicap psychique. Disponible sur www.unafam.org/Specificitede-I-handicap.html (consulté le 10 février 2014).

UNAPEI. Disponible Disponible sur www.unapei.org/ (consulté le 10 février 2014). 
lerPartie-T115 13/04/14 14:38 Page 30

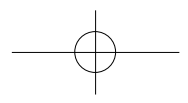

$30 \quad$ ] terminal $n^{\circ} 115[$
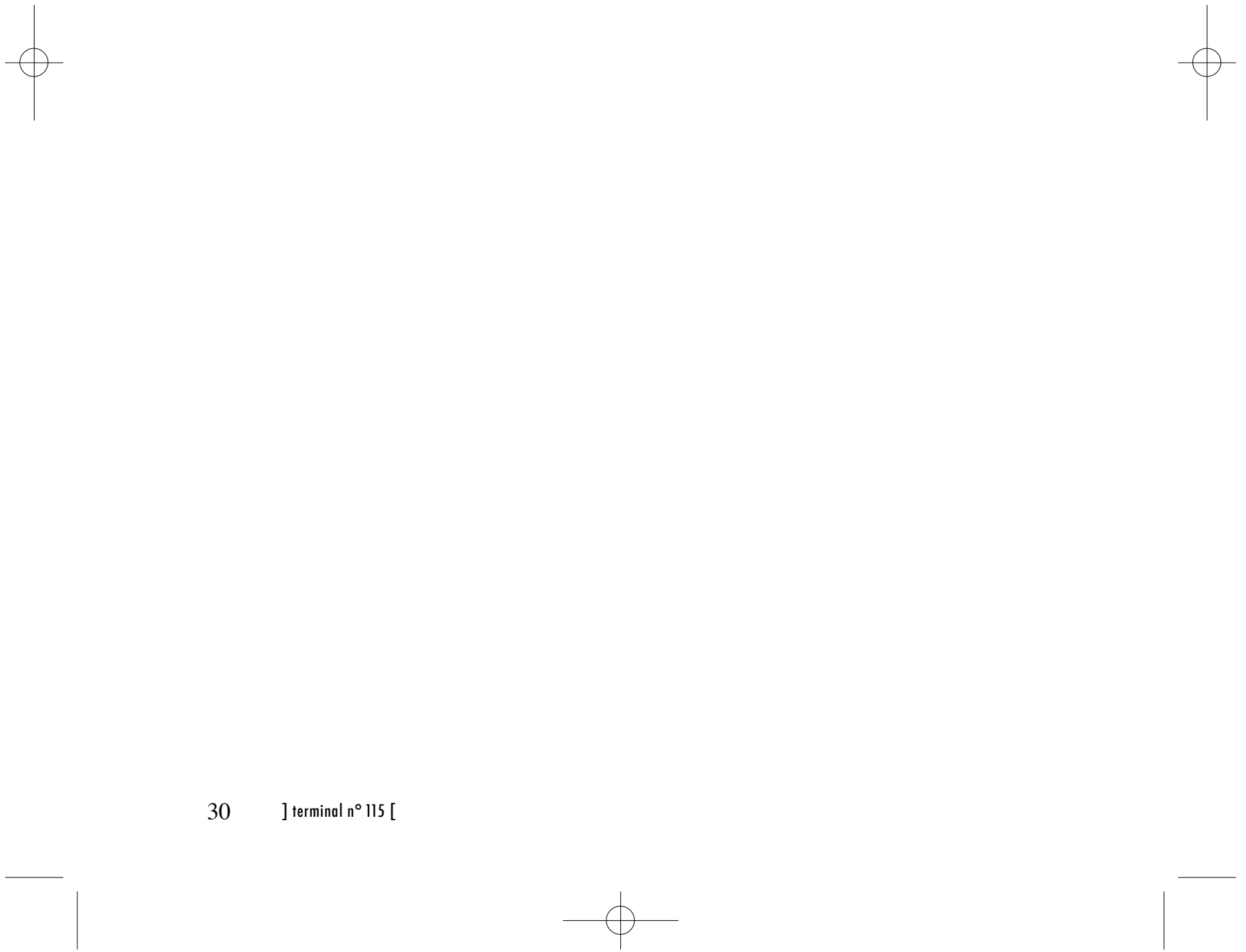